\title{
Estimating Common Pedestrian Routes through Indoor Path Networks using Position Traces
}

\author{
Thor S. Prentow, Henrik Blunck, Kaj Grønbæk, Mikkel B. Kjærgaard \\ Department of Computer Science \\ Aarhus University, Denmark \\ Email: prentow,blunck,kgronbak,mikkelbk at cs.au.dk
}

\begin{abstract}
Accurate information about how people commonly travel in a given large-scale building environment and which routes they take for given start and destination points is essential for applications such as indoor navigation, route prediction, and mobile work planning and logistics. In this paper, we propose methods for detecting commonly used routes by robust aggregation, clustering, and merging of indoor position traces. The developed methods overcome three specific challenges for detecting commonly used routes in an indoor setting based on position data: i) a high ratio between path-density and positioningaccuracy, ii) a flat path hierarchy, and iii) providing cost-effective scalability. Through an evaluation based on data collected by staff members at a hospital covering more than 10 hectare over three floors, we show that the proposed methods detect routes that are representative of the commonly used routes between locations. These methods are sufficiently efficient to provide common routes based on real-time data from thousands of devices simultaneously. Furthermore, we show that the methods operate robustly even on basis of noisy and coarse-grained position estimates as provided by large-scale deployable indoor $\mathrm{Wi}-\mathrm{Fi}$ positioning systems, and with no prior information on building layout.
\end{abstract}

\section{INTRODUCTION}

Accurate prediction of commonly used routes between pairs of start and end locations is essential for indoor applications such as navigation, travel-time estimation, facility planning and logistics. Route predictions can be performed without use of empirically collected data by residing to modeling and heuristics based on assumptions, e.g., that always the shortest or fastest paths are used. However, studies on indoor motion patterns performed by Millonig et. al. [1] show that the spatially shortest path is a poor predictor for the path used, as many factors influence the choice of path, such as the specific context and preferences of the person traveling. Similarly, a study performed by Letchner et. al. concludes that only $35 \%$ of outdoor travels follow the fastest path [2]. This suggests that in order to be accurate, route predictions must in part be performed based on empirical data on previously used routes, e.g. by detecting the commonly used routes. While methods exist for detection of commonly used routes for outdoor settings based on empirical data [3], [4], these cannot easily be applied to indoor environments due to their lack of addressing three indoor-specific challenges, which are further described in section II: i) An increase in the path-density versus positioning-accuracy, ii) a flattened path hierarchy, iii) providing cost-effective scalability.

In this paper, we propose methods for detecting commonly used routes by noise-resistant aggregation, clustering, and merging of indoor position traces. These methods form
InTraRoute, which given position traces from sensing mobile devices detects the commonly used routes between locations. The contributions of this paper include the three main components of which InTraRoute consists: The filtering component Direct Route Traversal Extraction, which facilitates unsupervised data-collection-from e.g. staff members performing their daily jobs - by filtering out indirect routes in collected position traces in order to extract only the direct routes between endpoints. The Efficient Route Clustering component which clusters position traces between two given endpoints and thereby allows for identification of the routes between theseof which there may be many, due to the flat path-hierarchy in indoor path networks. Finally, the Per Cluster Common Route Identification component, which identifies a representative of each cluster of route traversals, based on several potentially noisy position traces between two endpoints. Furthermore, we evaluate InTraRoute in regards to the representativeness of the identified common routes. The evaluation is performed using a large-scale position trace data set containing the movements of staff members at a large hospital covering more than 10 hectare split over three floors. The positions have been collected through Wi-Fi measurements from smart-phones carried by ten staff members during their daily work throughout ten days. Positions are estimated using two simple and scalable Wi-Fi positioning methods, with a mean positioning accuracy of $15 \mathrm{~m}$ and $30 \mathrm{~m}$, respectively. Through the evaluation, we show that the computed common routes are representative of the collected routes, even when the collected routes contain inaccurate outlier position estimates, and even when no prior knowledge of the building layout is available. Finally, we demonstrate that the methods are able to scale efficiently to a 10 hectare building infrastructure with 1359 detected locations connected by 13670 unique transitions, and that the methods will allow for real-time usage of more than 40.000 moving targets continually supplying data.

The methods proposed enable application types that depend on knowledge of common routes. These include indoor navigation, where new employees or visitors to buildings may be guided to use the routes commonly used by people often traversing the building, as well as route prediction, where the commonly used routes may serve as a good predictor of routes used. For facility management and analysis an overview of the commonly used routes can be used to identify and mitigate bottlenecks, e.g., to avoid blocking the preferred routes for maintenance reasons at critical hours [5]. Furthermore, the proposed methods enable automatic task scheduling systems as increasingly widespread, e.g. in hospitals, to plan tasks to be carried out in an order which allows for following the 
commonly used routes. In addition, automatic task scheduling requires precise predictions of the time involved in, e.g., traveling between work locations. The computation of these travel-time estimates may be greatly improved by accurate knowledge of the routes that will be used.

\section{INDOOR CHALLENGES}

In this section we further detail the three main indoorspecific challenges that prevent us from directly applying the available methods for outdoor common route detection.

High path-density and low positioning-accuracy: In an indoor environment, the ratio between path density and positioning accuracy is drastically increased: The path structure of a building consisting of hallways and rooms is much denser than the typical path structure of a road network. In addition, the accuracy of positioning systems which may easily be deployed in a large-scale building infrastructure is at best only as good as, and often worse than, GPS accuracy as experienced in outdoor scenarios. As a result, performing the identification and distinction of individual paths based on collected position traces is harder indoors, as the expected positioning errors may match or exceed the separation distance between route choices. Flat path hierarchy: Outdoor road networks form a hierarchy from freeways down to local gravel roads. Travelers can be expected to prefer paths high in this hierarchy. This in turn greatly reduces the number of plausible routes. In an indoor environment, however, these path hierarchies are much flatter, less distinct, and often chosen context-dependent-resulting in significantly more equally plausible routes for traveling between two given indoor locations.

Providing cost-effective scalability: In outdoor settings, satellite-based positioning and road-models are financed by national and regional funding and can be used by a comparatively large community of users, whereas in indoor environments the financial effort per user is usually larger and has to stem from the public or private owners. Therefore, methods for indoor route estimation have to be optimized for easy deployability, which sets specific limitations on the methods used: i) the availability of up-to-date digital building models cannot be assumed. These may unavailable or need time-consuming processing in order to extract walkable path networks. ii) advanced indoor positioning infrastructures cannot be assumed to be in place, as these may be very expensive to deploy for large building infrastructures; iii) time-consuming setup and maintenance such as up-to-date high-resolution fingerprinting cannot be assumed to be feasible for large buildings. [6]

\section{Common Route Detection}

In this section we describe the InTraRoute method for common route detection that has been developed in order to overcome the challenges described in section II. In the following, a position refers to a location in a building complex. A position estimate is the estimated timestamped position of a moving entity or target, as provided by an indoor positioning system. A position trace is a list of consecutive position estimates, collected as a specific target moves along routes through the covered building complex. Figure 1 provides an overview of the flow of data through InTraRoute, the individual steps of which are further detailed in the following subsections.

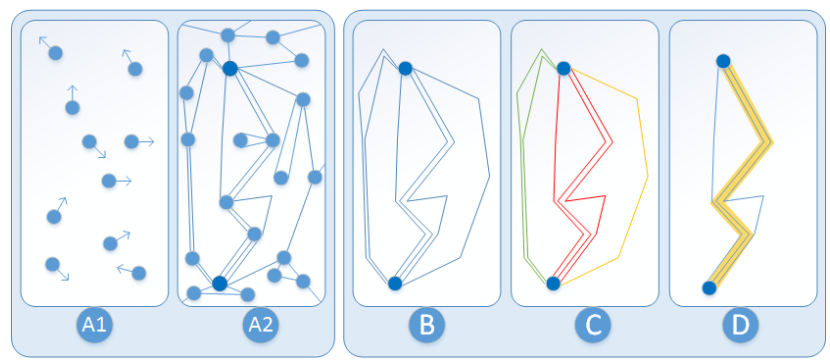

Fig. 1: The processing pipeline of InTraRoute. (A) Position Trace Collection. (B-D) InTraRoute: (B) Direct Route Traversal Extraction, (C) Efficient Route Clustering, (D) Per Cluster Common Route Identification

The targets for which common routes are to be extracted move around a building complex performing their daily routines, while an indoor positioning system computes position estimates (A1). For each individual target, a position trace consisting of its position estimates is maintained (A2). The traces are passed on to the InTraRoute method, the first step of which is the Direct Route Traversal Extraction module, which extracts from each trace the direct route traversals and removes the indirect route traversals. Furthermore, the traversals are grouped by their associated start-destination-pair of locations (B). The Efficient Route Clustering module clusters the direct route traversals for a given start and endpoint pair into clusters. Each such cluster is comprised of route traversals that are similar to each other, according to a noise resistant similarity measure (C). The Per Cluster Common Route Identification module finally aggregates the route traversals in the clusters containing most collected traversals in order to identify a route representative of the most common of the collected traversals for the given start-destination-pair (D).

\section{A. Position Trace Collection}

The input for InTraRoute is position estimates of moving targets, computed by a suitable indoor positioning system, e.g. Wi-Fi or Bluetooth. As such, the indoor positioning system and method is not considered part of InTraRoute. However, for completeness we briefly describe the indoor positioning method, which is used to evaluate InTraRoute in this paper: Mobile sensing devices are used to measure received signal strength of $\mathrm{Wi}-\mathrm{Fi}$ access points in the vicinity of the target device. These measurements are forwarded to a positioning server, which computes position estimates based on the measurement for each target. The positioning method employed is centroid lateration, as described in [7]. The method computes position estimates as the weighted average position of the received access points (AP), using the received signal strength (RSSI) as weight. The resulting position is mapped to the location of the nearest AP, in order to avoid position estimate outliers falling outside the building complex. This simple approach fulfills the goal of being easily scalable for deployment in a large building infrastructure, as the only infrastructure information required is the location coordinates of the APs. We have evaluated the Centroid Lateration method to yield a mean accuracy of $15 \mathrm{~m}$ in the investigated environment, whereas in comparison more advanced but less scalable Wi-Fi-based fingerprinting methods are expected to achieve accuracies down to a few meter [8]. Consecutive position estimates yielding the same location for a specific target are 
aggregated into intervals. The resulting position trace for an individual target consists of consecutive triples of the form $(T, I, P)$ containing a time-interval $T$, a target identifier $I$ and a position $P$.

\section{B. Direct Route Traversal Extraction}

Consider, for example, that the position traces are collected for a staff member at work. To identify commonly used routes from position traces, we aim to extract from each position trace the direct route traversals, i.e. the travels undertaken purposefully between two locations. We thus aim to identify start and end location (and times) of such direct travels, and to filter out indirect routes, i.e. route traversals containing detours, such as a toilet break or detour to the coffee machine. Filtering is performed in three ways: First, in order to reflect that direct route traversals should not contain loops, for each position trace which contains a loop of any length the trace is split into three parts: before loop, loop, and after loop; the loop part is considered an indirect travel and not processed further. Secondly, we also split position traces at locations, where the device remained stationary for more than a threshold period $T_{\text {stat }}$. The motivation is also here, that the stationary period signals that the target did not move consistently with purpose from a location before the stationary period to a location visited after the stationary period. Thirdly, we filter based on the stretch factor of routes traversals, as defined by Castro et al. [9]: We compute the stretch factor of a route traversal as the ratio of its length to the length of the 20th percentile of the recorded route traversals (w.r.t length) for the same start-destination-pair. To this end, length can be defined, e.g. in terms of travel time or travel distance. If the computed stretch factor is above a prescribed threshold $T_{\text {stretch }}$, the route traversal is discarded as indirect. When it is concluded that a target traveled directly from a start location to an end location, the respective route traversal data is used also as route traversal data for the sub-routes between the locations passed on the way. Thus, for each route traversal classified as direct, information on routes between several start-destination-pairs is extracted.

\section{Efficient Route Clustering}

The Route Clustering module groups the direct route traversals for each start-destination-pair into clusters of traversals following similar routes. The motivation for arranging route traversals into clusters is threefold: First, due to the flat path hierarchy two locations may have several commonly used routes between them. Second, some indirect traversals may remain, despite the Direct Route Traversal Extraction step, and thus may not stem from purposefully executed direct walks between a start-destination-pair. This module's clustering will separate these route traversals from commonly used routes, as the former will form smaller clusters. Thirdly, the low position accuracy relative to the high path density may lead to uses of the same commonly used route may produce slightly different route traversals. This module will allow distinguishing between different routes, while still being able to group together slightly dissimilar route traversals into the same cluster of routes. Using computationally efficient clustering aids to achieve scalability of InTraRoute compared to using more complex methods, e.g. the often-used suffix-tress, which in addition do not easily allow for handling noisy location data. We employ a similaritybased online clustering approach using a similarity measure $\operatorname{sim}\left(R_{1}, R_{2}\right)$ which computes the similarity between two route traversals $R_{1}$ and $R_{2}$. A measure of similarity $\operatorname{sim}(C, R)$ of a new route traversal $R$ to a cluster $C$ of route traversals is then obtained as the similarity $\operatorname{sim}\left(R_{c}, R\right)$ of $R$ to a representative route travel, representing the cluster $C$. This representative is available as the current result of InTraRoute's Per Cluster Common Route Identification module, described in detail in Section III.D. The newly collected route traversal $R$ is then either i) added to a most similar existing cluster of traversals or ii) used to create a new cluster if no clusters are sufficiently similar to the traversal, as decided by a similarity-threshold $T_{\text {sim }}$. Using an appropriate similarity threshold $T_{\text {sim }}$ yields that when using the same route, the resulting route traversals will be assigned to the same cluster, even if inaccuracies or noise in the Wi-Fi measurements or positioning occur. For suitably measuring similarity we considered as measures sim both spatial and temporal features of route traversals. Specifically, we will present results for using the following two measures: First, the Jaccard measure, defined as the relative number of locations in common between a new traversal $R$ and a cluster representative $R_{C}: \operatorname{sim}_{J}\left(R, R_{C}\right):=\frac{\left|R \cap R_{C}\right|}{\left|R \cup R_{C}\right|}$; and second, a travel time-based measure $\operatorname{sim}_{T}$, which is defined as the relative deviation of their travel times $t(R)$ and $t\left(R_{C}\right)$ : $\operatorname{sim}_{T}\left(R, R_{C}\right):=1-\left|\frac{t(R)-t\left(R_{C}\right)}{t\left(R_{C}\right)}\right|$. The latter measure puts a higher focus on clustering together routes of similar travel time, and produces common routes estimates that better reflect common travel times.

\section{Per Cluster Common Route Identification}

In this component, the route traversals for a given cluster $C$ of route traversals, computed as described in Section III-C, are merged into a single representative route. To this end, InTraRoute maintains as representation for each cluster a transition graph $G_{C}\left(V_{C}, E_{C}\right)$, where the nodes $V_{C}$ represent the set of locations contained in the route traversals that form the cluster, while the edges $E_{C}$ represent the position transitions in the route traversals in $C$. Each edge is annotated with the number of times the respective transition occurs in the route traversals in $\mathrm{C}$. These transition counts are maintained and updated each time a new traversal is added to a cluster (in the clustering module $(\mathrm{C})$ ): for each of the traversals' location transitions either i) the transition count for an existing edge is increased, or ii) a new edge is created if the edge did not exist in the cluster's transition graph. This counterannotated transition graph is now used to determine a commonroute representative for the respective cluster as follows: To each edge $e=\left(v_{1}, v_{2}\right)$ in $G_{C}$ a weight $W(e)$ is assigned as the squared inverse of its counter, i.e. of the number of route traversals in $C$ in which the transition $v_{1}$ to $v_{2}$ occurs. In effect, the most traversed edges will be given the lowest weights. This allows for common route extraction as a shortest path computation in the weighted Graph $G_{C}$. Such a shortest path can be computed using Dijkstra's algorithm (or variants thereof): As the most traversed edges are given the lowest weight, they will be chosen over less traversed edges. Thus, the computed route will utilize, as desired, foremost the most commonly traversed edges in the graph while at the same time utilizing not excessively many edges in total. 


\section{EVALUATION}

We evaluate InTraRoute based on the representativeness of detected common routes, the dependence on positioning method as well as the scalability to large building infrastructures. This is done based on a dataset collected at a large public hospital, covering more than $100.000 \mathrm{~m}^{2}$ over three floors. Data was collected using Google Nexus 4 smartphones. The phones ran sensor-logging software to log Wi-Fi signal strengths with a sampling rate of approximately $0.5 \mathrm{~Hz}$. Ten smartphones were provided to the maintenance staff of the hospital. These phones were carried through their regular work, during both day, evening and night shifts, for ten consecutive days except when charging. In total, this collection procedure produced the equivalent of 312 hours of continual position traces. Collecting positioning ground truth for this dataset was prohibitive due to the large scale of the experiment in both spatial extent of the environment and duration, as well as due to privacy concerns restricting the use of e.g. video recording [5]. In addition, we perform an initial evaluation using a dataset from a constrained area, which allows us to collect ground truth. In the latter dataset, 3 different routes between a specific start and end point were traversed for a total of 18 traversals.

\section{A. Constrained Area Evaluation}

We first provide an initial evaluation of individual steps in the InTraRoute method, both in order to give further support for their usage as well as to provide better intuition of them. We do so using the dataset from a constrained area of the hospital. The three different routes between a given pair of endpoints are depicted in Figure 2a. Ten traces were collected following the red route, six following the green and two following the yellow one. Figure $2 \mathrm{~b}$ shows the result after the Efficient Route Clustering step, i.e. the transition graph $G_{C}$ for each of the detected clusters, wherein each edge is color-annotated by the cluster into which its represented transition falls. Individual examination of the traversals reveals that evidently all route traversals are associated with the correct one among the three clusters. Additionally, the figure illustrates the position estimate outliers that may occur, indicated by positions being far from the route used, as a result of the simple centroid-lateration positioning method used. Figure $2 \mathrm{c}$ shows the result of the Per Cluster Common Route Identification step performed for each cluster. It shows that the final route traversals closely resemble those routes actually used, and that the position estimate outliers have been filtered out successfully. To further motivate the clustering approach, the white line in Figure $2 \mathrm{c}$ illustrates the route resulting from merging all traversals without prior clustering. While this route seems reasonable, it never actually occurs in the traversals but is instead a combination of the two most common routes, which are correctly identified as such by the clustering module. To conclude, the initial exploratory investigation illustrated by Figure 2c suggests that InTraRoute, and its individual components, work as intended.

\section{B. Representativeness of Routes}

To obtain a measure for the representativeness of a InTraRoute-computed common route representative $R_{C}$ for a cluster $C$ of route traversals for a given start-destinationpair, we make use of the similarity measures $\tilde{\operatorname{sim}}(C, R)$ as introduced in Section III-C, concretely of the Jaccard measure and the travel time-based measure: We define for $R_{C}$ its representativeness $r_{\text {sim }}\left(R_{C}\right)$ as the average similarity it has with the route traversals in $C$. By extension, we obtain also a global similarity measure for the InTraRoute-computed common route representatives over a given dataset, by averaging $r_{\text {sim }}\left(R_{C}\right)$ over all detected route clusters $R_{C}$. We though decided to exclude from the global measure those (route clusters for) start-destination-pairs, which have no traversals between them with at least 4 transitions: These short traversals are excessive in numbers and would skew the global measure to provide overly optimistic results-because common routes are easier to compute for such very short routes. Based on the global measure, Figures $3 \mathrm{a}$ and $3 \mathrm{~b}$ show the representativeness of the InTraRoute-computed representative routes as a progression when increasingly larger portions of the main data set are used: representativeness is measured on basis of the Jaccardmeasure in Figure 3a, and the travel-time-based measure in 3b. Each of the two figures show graphs for in total five different methods of calculating representative routes: Shown are the two different similarity measures used in the clustering step of InTraRoute, the Jaccard-measure (red), and the travel-timebased measure (green). For comparison, graphs are given also for two simple alternative methods for selecting representatives of common routes between start-destination-pairs: selecting the fastest (blue) and the shortest (pink) detected route traversal, respectively. Finally, the violet graph shows the average pairwise similarity for route traversals between a start-destinationpair, averaged over the set of all start-destination-pairs.

The data shown in both Figure $3 \mathrm{a}$ and Figure $3 \mathrm{~b}$ illustrates that InTraRoute detects common routes that are more representative of the collected route traversals than the ones produced by the comparatively simpler methods. Furthermore, it shows that in InTraRoute the Jaccard-based clustering results in representativeness slightly superior to the travel-time-based similarity measure for clustering-when measuring representativeness based on the Jaccard-measure. Figure 3 a also shows that the pair-wise similarity of collected traversals decreases as more data is collected, due to the more diverse situations in which position traces are collected as time progress, e.g. by different staff members, week days and locations (which also explains the outliers in the progression of the graphs). The fact that InTraRoute continues to produce representative common routes-despite this decrease in route traversal equalitymotivates its use, as does comparing the absolute similarity to other graphs in Figure 3a: The average Jaccard-similarity for a computed common route w.r.t. the route traversals from which they are computed is significantly higher than the average similarity between two original route traversals.

In Figure 3b, i.e. when comparing using the travel-timebased measure, the superiority of the two InTraRoute variants is even more pronounced: The resulting relative travel time deviations converge for increasing data amounts to only ca. $10 \%$, while the deviations for simpler alternatives converge to ca. $40 \%$. Furthermore, deviations produced in InTraRoute are lower when using the time-based instead of the Jaccardbased measure for clustering. This indicates that the former should be used if the system is not used primarily for detecting commonly used routes, but instead for providing travel time estimates between given start and destination locations. 

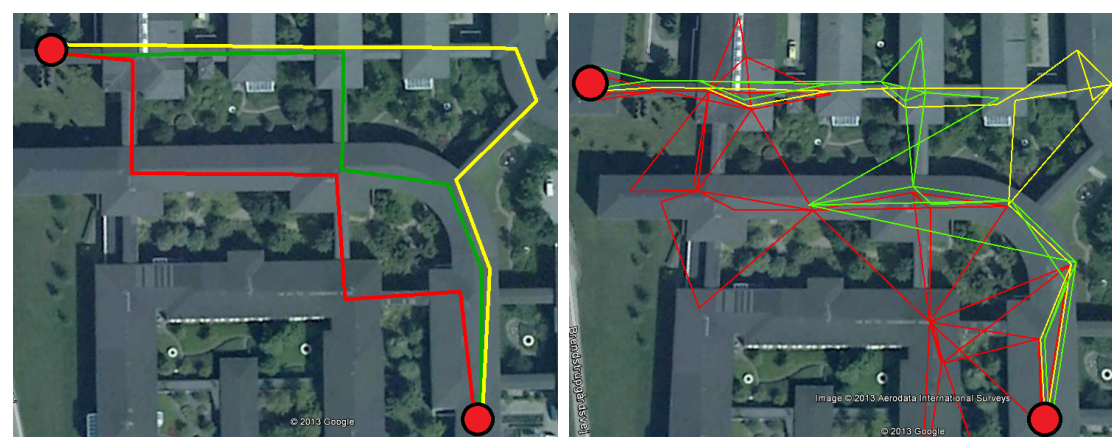

(a) Ground truth for routes traversed. The (b) Transition graph. Each edge is colored red route was traversed 10 times, the green according to which cluster it belongs to 6 times and the yellow 2 times. (following the step Efficient Route Clustering step).

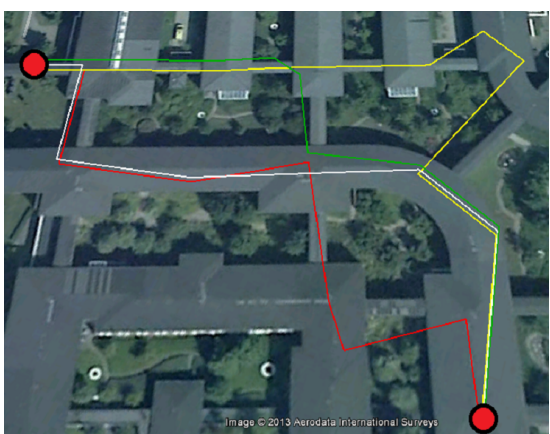

(c) The common routes computed in the Common Route Identification step: for each cluster (colored) and for merging all route traversals (white) with no clustering.

Fig. 2: Visualizations of the route data set discussed in Section IV-A: Ground truth (a), transitions graph (b) and identified common routes (c). Map data: Google, Aerodata International Surveys

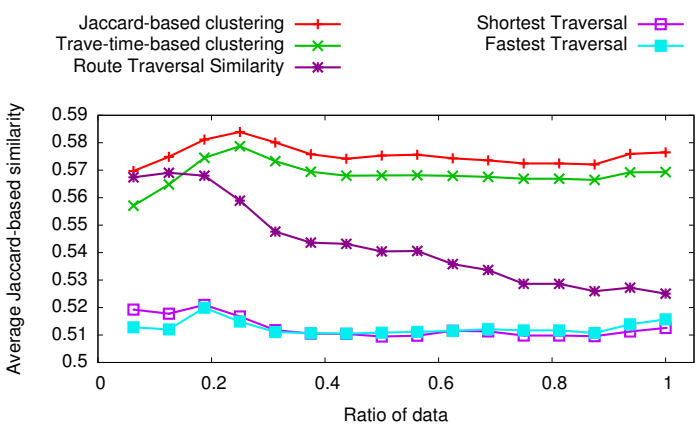

(a) Resulting similarities w.r.t. the Jaccard measure.

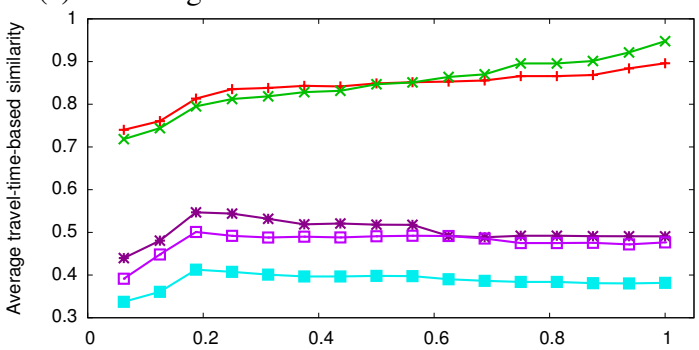

(b) Resulting similarities w.r.t. the travel-time-based measure.

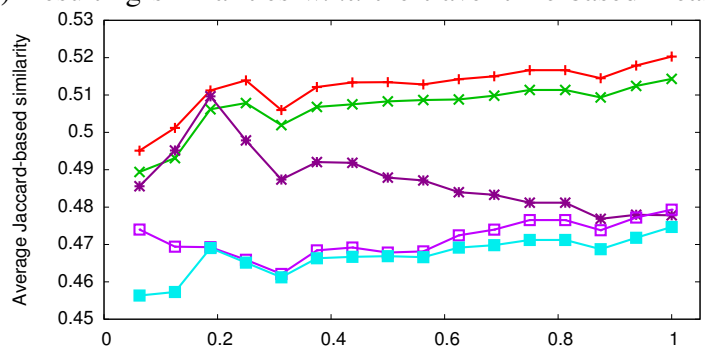

(c) Results as in (a) but using the strongest AP positioning instead of the centroid positioning.

Fig. 3: Similarity progressions growing amounts of input data when using different measures to compute similar routes

\section{Dependence on Positioning Method}

To evaluate the dependence on the positioning method used and on its available accuracy, we for comparison in Figure 3c recomputed the results from Figure 3a but instead using the simpler strongest AP positioning method, which was evaluated to have a mean accuracy of $30 \mathrm{~m}$ at the deployment site. This

\begin{tabular}{|r||c|c|c|c|c|}
\hline Data Size & $1 / 16$ & $1 / 8$ & $1 / 4$ & $1 / 2$ & 1 \\
\hline Locations & 827 & 1050 & 1206 & 1297 & 1359 \\
\hline Edges & 3845 & 6171 & 8777 & 11022 & 13670 \\
\hline Transitions & 24428 & 60369 & 133181 & 294392 & 560347 \\
\hline Time (MM:SS) & $00: 14$ & $00: 34$ & $01: 12$ & $02: 40$ & $05: 22$ \\
\hline
\end{tabular}

TABLE I: Statistics for the increasingly larger portions of the dataset that are used in the evaluation.

method simply estimates the position of a target to be the location of the AP for which it receives the strongest RSSI. We see that the progression with increasing amounts of realworld input data is highly similar to that which occurred when using the centroid-lateration approach. The absolute similarities achieved are though somewhat lower than those given in Figure 3a. This suggests that the InTraRoute method still performs robustly and reasonably well also when imprecise positioning data is used as input-but InTraRoute suffers also to some extent from a decrease in positioning accuracy. This decrease though seems likely to be unavoidable as it is inherent in the collected trace data: We see that also the overall similarity between route traversals is lowered in equal manner, from 0.52 to 0.47 , due to the decrease in positioning accuracy.

\section{Scalability}

In order to evaluate the efficiency and scalability of the system with regards to amounts of data to be analyzed, runtime was measured for increasingly larger amounts of data in the large building infrastructure. Table I shows the results from running the system on increasingly larger portions of the complete dataset. Visible from the table is, as expected, a quasi-linear relation between the amount of processed position traces and runtime. This provides evidence that the system can easily accommodate large amounts of data. Analysis of the entire dataset of 312 hours of position traces takes 5 minutes, which suggests that the system is able to keep up with real-time data in even the largest and most frequented building complexes. As all methods in the system are built for an online approach, new data can be added as it is collected without the necessity of recomputing large amounts of data. Indeed the time used to process each new position estimate is measured to be on average below 0.05 millisecond, which would theoretically allow for concurrent updates from more than 40.000 moving targets each supplying position updates at $0.5 \mathrm{~Hz}$. In addition, the table shows that the number of different 
locations rises rapidly as data is added at first, but then seems to converge. This is as is expected when positioning at a large indoor infrastructure, as at some point most or all locations will have been detected. It also means that the graph-structure of our methods do not grow indefinitely, but at some point only e.g. the traversal counts of the cluster graphs will increase no matter the amount of data added from the same infrastructure. We see the same slow down in the amount of new edges, just in larger numbers. However, the number of transitions occurring as expected increases steadily.

\section{RELATED WORK}

Various methods have been developed for automating indoor map construction and detection of building layout, e.g. via depth camera [10], inertial sensors and landmark detection [11], or based on Wi-Fi fingerprints [12]. While these methods help in constructing a floor plan when none is available, they come with further requirements such as precise positioning and specialized hardware. For detecting common routes without depending on a map, e.g. Radaelli et al. [13] present techniques for identifying typical movements among moving targets using a bluetooth positioning system. For outdoor settings a lot of methods have been developed for route prediction based on GPS devices, e.g. to precisely estimate time-of-arrival [4], for mining routes commonly used by taxi drivers [14], or to navigation based on detected personal preferences [2]. Common for these approaches are that they require the availability of a road map, to which they either snap the GPS positions or use for routing. Similar to indoor, methods are also available for automatic construction of road maps, e.g. based on GPS traces from buses [3], or based on intersection detection [15]. Common for these and similar methods are that they rely on the low path-density versus positioning-accuracy ratio when utilizing the GPS system for low-density road-networks compared to indoor paths. For indoor positioning [16] several techniques have been proposed which offer accuracies similar to or better than obtainable by GPS in an indoor context, but which require an investment in expensive infrastructure [17], or time-consuming fingerprinting of the entire environment [8].

\section{CONCLUSION}

In this paper, we proposed new methods for detecting commonly used routes for indoor building infrastructures based on empirical data from simple positioning systems, with no prior knowledge of building layout. Through evaluations using datasets collected at a large hospital, we show how the methods produce routes that are representative of the commonly used routes in the building. In addition, we show that the methods are able to scale to a large building infrastructure. The methods thereby successfully overcome the three challenges we describe for performing indoor common route detection: high path-density to positioning-accuracy ratio, a flat path hierarchy and cost-effective scalability. The InTraRoute method may be used for applications such as building navigation, route prediction, facility management and automatic task scheduling at large building infrastructures. The system has opportunities for further improvement by extending the individual techniques and adding further functionality. This includes more complex noise filtering and smoothing approaches based also on e.g. duration, or more advanced similarity measures, e.g. a combination of the two used measures, or adapted measures for string similarity. The trade-off to be decided on though is how much efficiency to sacrifice for more advanced methods.

\section{ACKNOWLEDGMENT}

We thank the employees of Aarhus University Hospital, Skejby, as well as Systematic for their help in data collection. The authors acknowledge the support granted by the Danish Advanced Technology Foundation under J.nr. 076-2011-3.

\section{REFERENCES}

[1] A. Millonig and G. Gartner, "Identifying motion and interest patterns of shoppers for developing personalised wayfinding tools," J. Location Based Services, vol. 5, no. 1, pp. 3-21, 2011.

[2] J. Letchner, J. Krumm, and E. Horvitz, "Trip router with individualized preferences (trip): Incorporating personalization into route planning," in Proceedings of the National Conference on Artificial Intelligence, vol. 21, no. 2, 2006, p. 1795.

[3] J. Biagioni, T. Gerlich, T. Merrifield, and J. Eriksson, "Easytracker: automatic transit tracking, mapping, and arrival time prediction using smartphones," in Proceedings of the 9th ACM Conference on Embedded Networked Sensor Systems, 2011.

[4] A. Karbassi and M. Barth, "Vehicle route prediction and time of arrival estimation techniques for improved transportation system management," in Intelligent Vehicles Symposium, 2003. Proceedings. IEEE, 2003, pp. 511-516

[5] A. Ruiz-Ruiz, H. Blunck, T. S. Prentow, A. Stisen, and M. B. Kjærgaard, "Analysis methods for extracting knowledge from large-scale wifi monitoring to inform building facility planning," in PerCom '14, 2014.

[6] M. B. Kjærgaard, M. V. Krarup, A. Stisen, T. S. Prentow, H. Blunck, K. Grønbæk, and C. S. Jensen, "Indoor positioning using wi-fi-how well is the problem understood?" in International Conference on Indoor Positioning and Indoor Navigation, vol. 28, 2013, p. 31st.

[7] A. LaMarca, Y. Chawathe, S. Consolvo, J. Hightower, I. Smith, J. Scott, T. Sohn, J. Howard, J. Hughes, F. Potter et al., "Place lab: Device positioning using radio beacons in the wild," in Pervasive Computing, 2005, pp. 116-133.

[8] P. Bahl and V. Padmanabhan, "Radar: an in-building rf-based user location and tracking system," in INFOCOM 2000. Nineteenth Annual Joint Conference of the IEEE Computer and Communications Societies. Proceedings. IEEE, vol. 2, 2000, pp. 775-784.

[9] P. S. Castro, D. Zhang, and S. Li, "Urban Traffic Modelling and Prediction Using Large Scale Taxi GPS Traces,” pp. 57-72, 2012.

[10] H. Du, P. Henry, X. Ren, M. Cheng, D. B. Goldman, S. M. Seitz, and D. Fox, "Interactive $3 \mathrm{~d}$ modeling of indoor environments with a consumer depth camera," in UbiComp. ACM, 2011, pp. 75-84.

[11] M. Alzantot and M. Youssef, "Crowdinside: automatic construction of indoor floorplans," in SIGSPATIAL/GIS. ACM, 2012, pp. 99-108.

[12] Y. Jiang, Y. Xiang, X. Pan, K. Li, Q. Lv, R. P. Dick, L. Shang, and M. Hannigan, "Hallway based automatic indoor floorplan construction using room fingerprints," in UbiComp. ACM, 2013, pp. 315-324.

[13] L. Radaelli, D. Sabonis, H. Lu, and C. S. Jensen, "Identifying typical movements among indoor objects - concepts and empirical study," in International Conference on Mobile Data Management.

[14] J. Yuan, Y. Zheng, C. Zhang, W. Xie, X. Xie, G. Sun, and Y. Huang, "T-drive: driving directions based on taxi trajectories," in Proceedings of the 18th SIGSPATIAL International Conference on Advances in Geographic Information Systems, ser. GIS '10, 2010, pp. 99-108.

[15] A. Fathi and J. Krumm, "Detecting road intersections from gps traces," in Geographic Information Science. Springer, 2010, p. 5669.

[16] M. B. Kjærgaard, "Indoor location fingerprinting with heterogeneous clients," Pervasive and Mobile Computing, vol. 7, no. 1, pp. 31 - 43, 2011.

[17] A. Ward, A. Jones, and A. Hopper, "A new location technique for the active office," Personal Communications, IEEE, vol. 4, no. 5, pp. 42-47, 1997. 